Original Research Paper

\title{
Characterisations of Parameters of Granularity of Sediments from Togolese Littoral: Granular Potential
}

\author{
Amey Kossi Bollanigni and Neglo Kouma \\ Ecole Nationale Supérieure d'Ingénieurs, Université de Lomé, BP: 1515 Lomé, Togo
}

\section{Article history}

Received: 11-07-2018

Revised: 14-07-2018

Accepted: 10-10-2018

Corresponding Author: Amey Kossi Bollanigni Ecole Nationale Supérieure d'Ingénieurs, Université de Lomé, BP: 1515 Lomé, Togo E-mail: ameykoss3@yahoo.fr ameyarticl@yahoo.com

\begin{abstract}
The present study aims at analysing the dimensional distribution of granulate grains all along the Togolese littoral and identifying the law of their longitudinal distributions. In this regard two hundred and ten (210) samples are collected all along the Togolese littoral from the Togo-Ghana border up to Togo-Benin border and another ten (10) samples on each side of a spike located at $37.5 \mathrm{PK}$. Samples dried at $105^{\circ} \mathrm{C}$ for $20 \mathrm{~h}$ are then subjected to screening test. Their longitudinal size distributions, uniformity coefficients and bendings are then specified. It is apparent that the sediments of the Togolese littoral consist of grains of category ' $F_{3}$ " that are getting bigger and bigger, less and less tight, badly graduated and more open in accretion and erosion zones in the direction of the littoral drift. Natural and artificial works all along the Togolese littoral enable to identify three categories of sediment (fine, medium-size and big) corresponding to properly determined usages in the concrete.
\end{abstract}

Keywords: Sediment, Togolese Littoral, Longitudinal Distribution, Size Distribution

\section{Introduction}

Most of civil engineering works, completed in Togo are in concrete which is a composite material made generally of granulates, binders and water. The choice of the size of granulates depends on expected results for concrete (desired features) such as Watertight concrete, shockproof concrete, hard wearing, compression resistant, bending resistant and impact resistant concrete, permeable concrete, workability of concrete: These are the features that are found for a concrete (Aitcin, 1998). The fundamental perimeter that influences the physical aspect of concrete is the granulate form characterized by its size distribution.

Various types of sands are used in Togo as granulates: Sea sands, river sands, sands from crushing of rocks and continental dunes. In order to meet sand demands projects are completed in Lomé and in the Togolese littoral areas. In the course of these projects sands from the Togolese littoral are very often used without knowing their different characteristics depending on their transversally and longitudinally geographical position.

The aim of this study is to find out the law of the size and longitudinal distribution through parameters of differential distribution, coefficients of Hazen, uniformity and granulate class of the littoral sediments of Togo. The determination of granulate properties of sandy sediments is being searched on the samples all along the Togolese littoral on $50 \mathrm{~km}$ from the border with Ghana (PK0) up to Benin border (PK50) in order to specify the potentials of each profile in granulates for concrete. Which will enable the optimization of this material in the civil engineering construction and therefore the optimization of the extraction of these sandy sediments.

\section{Basic Equations}

The typical, cumulative standard distribution $Q_{r}(x)$ of a distribution illustrated through Fig. 1 lies between 0 and 1 , i.e., between $0 \%$ and $100 \%$. Its expression is given by the following equation (NF ISO 9276-1, 1998):

$$
Q_{r, i}=\sum_{v=1}^{i} \Delta Q_{R, v}=\sum_{v=1}^{i} \bar{q}_{r, v} \Delta x_{v}
$$

With $1 \leq v \leq i \leq n$.

The differential distribution $q_{r}(x)$ (Fig. 1) is obtained by the equation:

$$
q_{r}(x)=\frac{d Q_{r}(x)}{d x}
$$




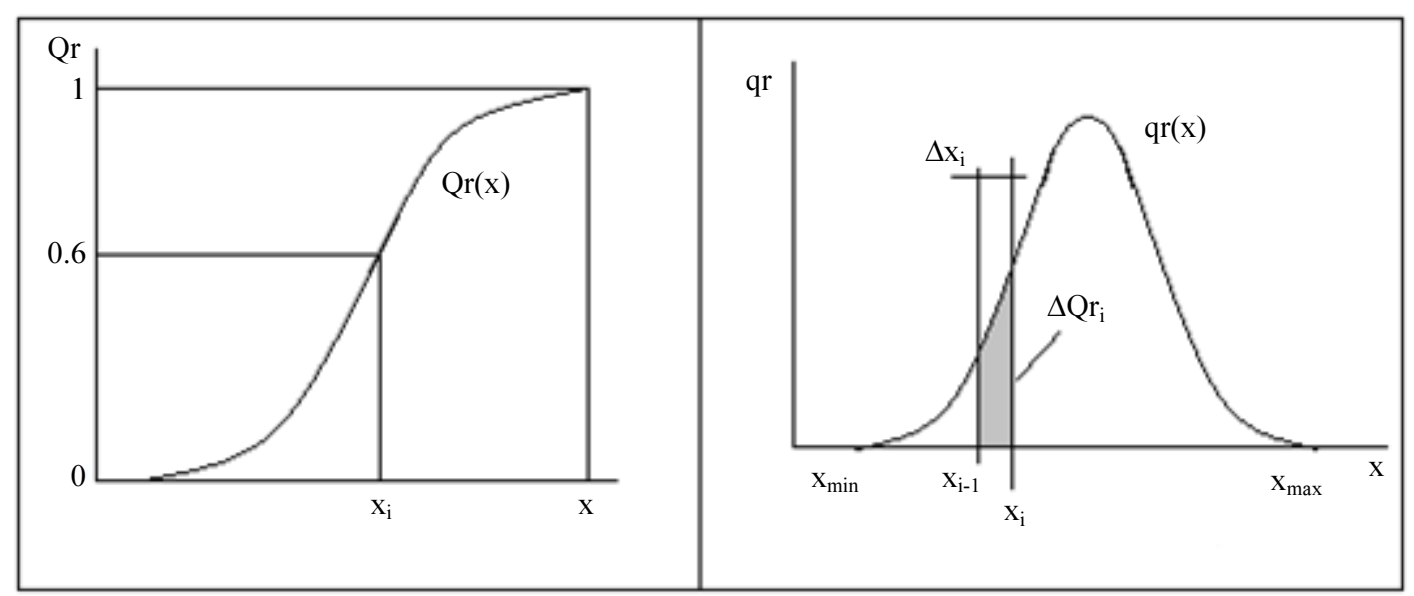

(a)

(b)

Fig. 1: Curves of cumulative and differential distributions (NF ISO 9276-1, 1998) (a) Cumulative distribution Qr(x) (b) Differential distribution $\mathrm{qr}(\mathrm{x})$

Table 1: Classification of materials according to coefficients of Hazen (of uniformity)

\begin{tabular}{ll}
\hline Nature of soil & $\mathrm{Value}$ of $\mathrm{Cu}$ \\
\hline Material with a highly tight grain size & $\mathrm{Cu}<2$ \\
Material with a tight grain size & $2 \leq \mathrm{Cu}<5$ \\
Material with a highly semi-tight grain size & $5 \leq \mathrm{Cu}<20$ \\
Material with a stretched grain size & $20 \leq \mathrm{Cu}<200$ \\
Material with a highly stretched grain size & $\mathrm{Cu} \geq 2$ \\
\hline
\end{tabular}

Table 2: Identification of the graduation of materials

\begin{tabular}{ll}
\hline Nature of soil & Value of Cc \\
\hline Case of gravel & $\mathrm{Cu}>4$ and $1<\mathrm{Cc}<3$ \\
Well graduated and clean material (GW) & $\mathrm{Cu} \leq 4$ or $\mathrm{Cc} \leq 1$ or $\mathrm{Cc} \geq 3$ \\
Badly graduated material (GP) & \\
Case of sand & $\mathrm{Cu}>6$ and $1<\mathrm{Cc}<3$ \\
Well graduated and clean material (SW) & $\mathrm{Cu} \leq 6$ or $\mathrm{Cc} \leq 1$ or $\mathrm{Cc} \geq 3$ \\
\hline
\end{tabular}

The evaluation of the granular surface area can be measured by coefficients of uniformity $\mathrm{Cu}$ and curve $\mathrm{Cc}$ the expressions of which are given by (Tchouani Nana and Callaud, 2004):

$$
\begin{aligned}
& C u=\frac{x_{60}}{x_{10}} \\
& C c=\frac{\left(x_{30}\right)^{2}}{x_{10} \times x_{60}}
\end{aligned}
$$

The parameters of these two equations are defined by:

$x_{60}$ : The screening correspondent to $\mathrm{qr}_{60}$

$x_{30}$ : The screening correspondent to $\mathrm{qr}_{30}$

$x_{10}$ : the screening correspondent to $\mathrm{qr}_{10}$

Following the values of $\mathrm{Cu}$ et $\mathrm{Cc}$ the sediments can be classified according to Table 1 and 2 .

The granulates are also charactrised by the diameter of the smallest grain « $\mathrm{d} »$ and that of the biggest grain «
$\mathrm{D} \gg$ and are expressed by $\mathrm{d} / \mathrm{D}$ and called granular class of a granulate. For sands, given the very small value of $\mathrm{d}$, the NF standard recommends to write 0/D (NF EN 12620, 2002) (Bresson, 1996).

In practice, this condition is seldom observed. The standard has then required criteria of acceptation of a granulate. For a superposition of sieve of stitch 1.56D-D$\mathrm{d}$ and $0.63 \mathrm{~d}$, this criteria is expressed by the system of the following equations (Amey, 2006):

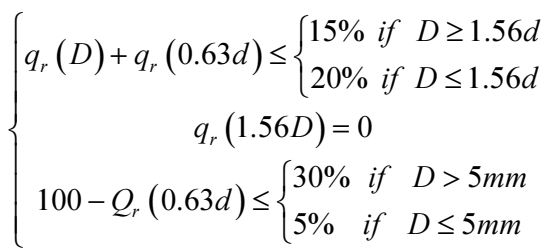

\section{Materials and Methods}

Collections of samples of sediment are carried out on thirty-five (35) profiles from the border Togo - 
Ghana up to Togo - Bénin (Fig. 2). Table 3 shows the numbers of profiles that are surveyed as well as their distance with regard to the border with Ghana (PK).

On each of the profiles, sediments are collected on the low-foreshore (Bas-E), the semi- foreshore (Mi-
E), the high-foreshore (Haut-E) and on the aerial beach at $5 \mathrm{~m}$ of high- foreshore (Début -PA), $10 \mathrm{~m}$ the high -foreshore (Mi-PA) and at the end of aerial beach (Fin-PA) as shown on Fig. 3.

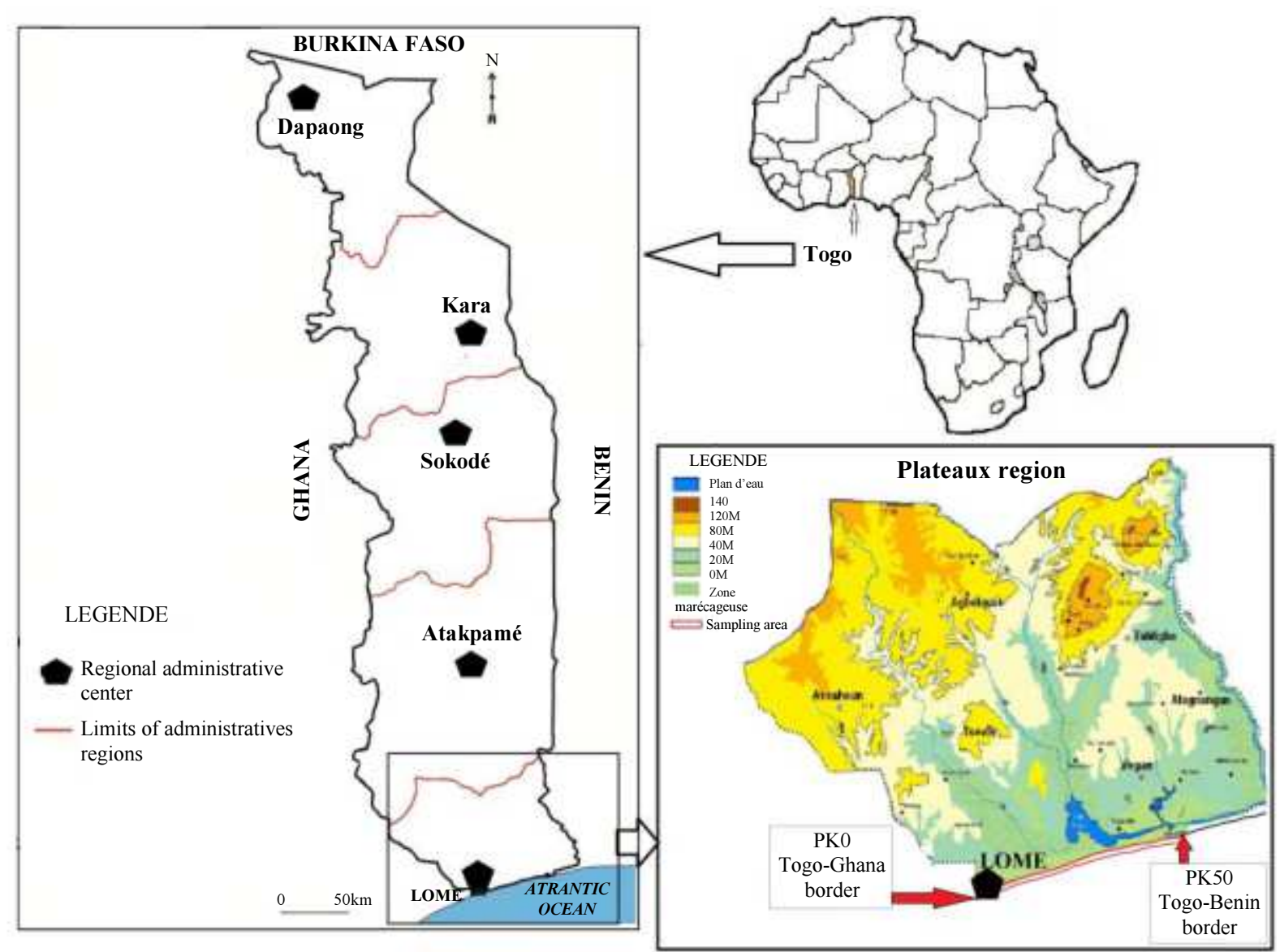

Fig. 2: Location of Togolese beach

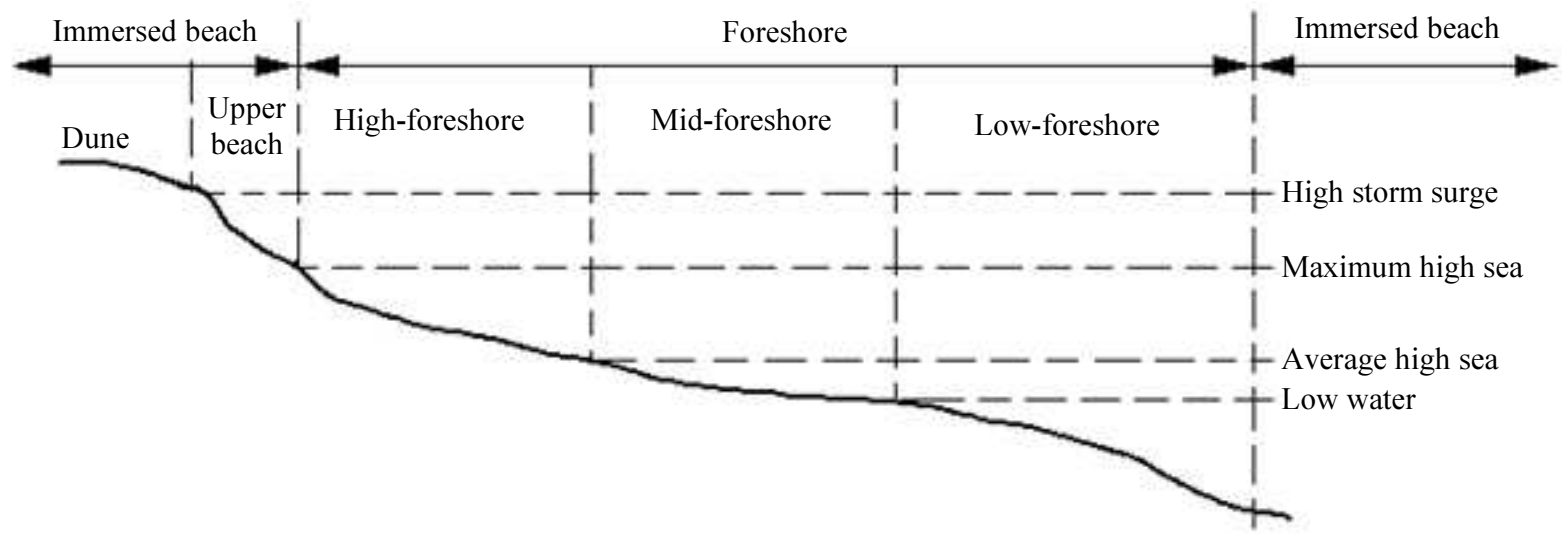

Fig. 3: Profile through a beach showing the foreshores and the aerial beach 


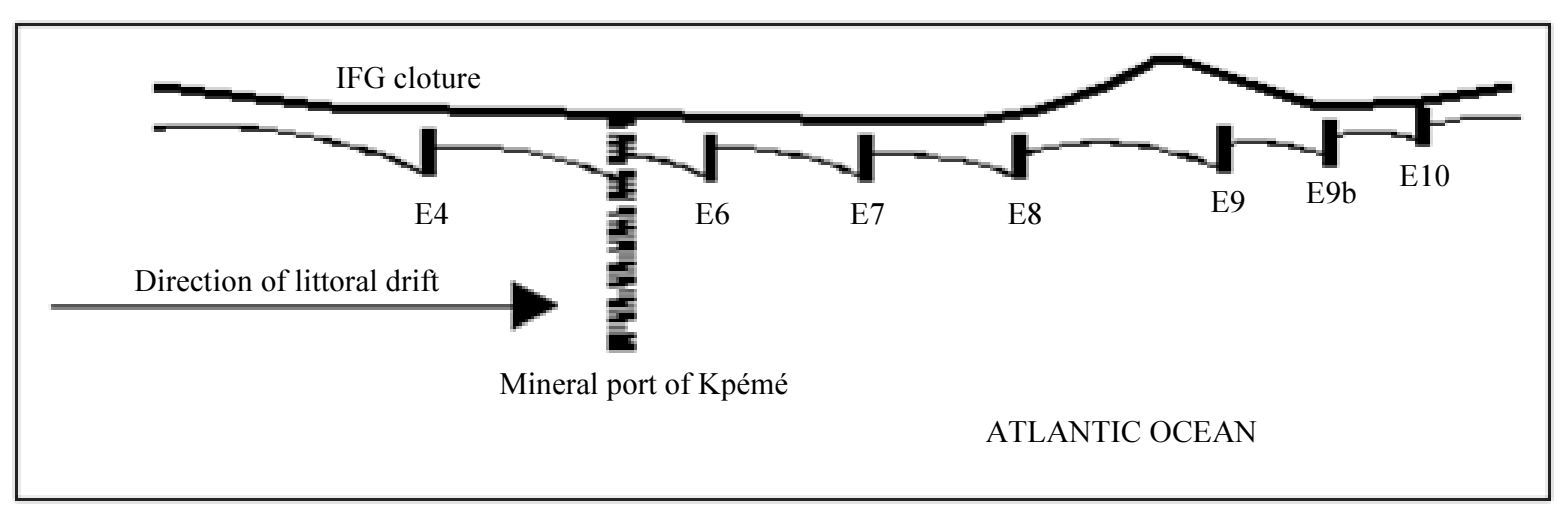

Fig. 4: Positions of the spikes on the coast

Table 3: Positions of the different profiles

\begin{tabular}{|c|c|c|c|c|c|c|c|c|c|c|c|c|c|c|c|c|c|c|}
\hline Profile & 1 & 2 & 3 & 4 & 5 & 6 & 7 & 8 & 9 & 10 & 11 & 12 & 13 & 14 & 15 & 16 & 17 & 18 \\
\hline Distance & 0 & 2 & 4 & 5 & 6 & 7 & 8 & 0 & 10 & 12 & 16 & 18 & 20 & 24 & 28 & 29 & 30 & 32 \\
\hline Profile & 19 & 20 & 21 & 22 & 23 & 2 & 25 & 26 & 2 & 28 & 29 & 31 & 31 & 32 & 33 & 34 & 35 & \\
\hline Distance: PK (km) & 34 & 35 & 36 & 37 & 38 & 39 & 40 & 41 & 42 & 43 & 44 & 45 & 46 & 47 & 48 & 49 & 50 & \\
\hline
\end{tabular}

Table 4: Positions of profiles with regard to the spike E4

\begin{tabular}{|c|c|c|c|c|c|c|c|c|c|c|}
\hline \multirow{2}{*}{$\begin{array}{l}\text { Positions } \\
\text { Profile }\end{array}$} & \multicolumn{5}{|c|}{ Western of the spike E4 } & \multicolumn{5}{|c|}{ Eastern of the spike E4 } \\
\hline & 1 & 2 & 3 & 4 & 5 & 6 & 7 & 8 & 9 & 10 \\
\hline Distances with regard to the spike (m) & 400 & 300 & 200 & 100 & 10 & 10 & 100 & 200 & 300 & 400 \\
\hline
\end{tabular}

Ten (10) other collections are carried out on the low foreshore on each side of the spike E4 located near PK37.5 (Table 4 and Fig. 4) of following characteristics:

- Glassy coastline: $-0.01 \mathrm{~m}$

- Length: $70 \mathrm{~m}$

The collected sands are dried in a proofer at a temperature of $105^{\circ} \mathrm{C}$ for $20 \mathrm{~h}$ (NF EN 933-1, 1997). The dried samples are subject to a test of sifting on the stitch sieves $0.08 \mathrm{~mm}-0.125 \mathrm{~mm}-0.25 \mathrm{~mm}-0.5 \mathrm{~mm}$ $1 \mathrm{~mm}-2 \mathrm{~mm}-4 \mathrm{~mm}$ et $5 \mathrm{~mm}$ (NF EN 933-2, 1996).

And then, the granular parameters are determined: size distribution, the coefficients of Hazen and uniformity and the granular class

\section{Results}

\section{Longitudinal Differential Distribution}

The Fig. 5 and 6 show the longitudinal grain-size distributions of sand all along the Togolese littoral and on each side of the spike E4. The differential distributions are the average of the samples of the 6 points of collection by profile.

From the curves on Fig. 5 and 6 it is apparent that:

- The sea sand contains practically no grains under 0.125 $\mathrm{mm}$ and over $4 \mathrm{~mm}$ (proportions under 1\%) (Fig. 5)
- The grains of $0.5 \mathrm{~mm}, 1 \mathrm{~mm}, 2 \mathrm{~mm}$ et $4 \mathrm{~mm}$ are generally of growing proportions of PK0 (profile 1) at PK 50 (profile 35). However, the grains of 0.125 et $0,25 \mathrm{~mm}$ present an average differential distribution $q_{r m}$ decreasing (Fig. 5)

The equations from the smooth curves that illustrate these behaviours are given by:

$$
\begin{aligned}
& q_{r<0.08}(x)=0.0015 x+0.0035 \\
& q_{r 0.08}(x)=0.0052 x+0.1424 \\
& q_{r 0.125}(x)=-0.1031 x+7.3247 \\
& q_{r 0.25}(x)=-0.1064 x+43.502 \\
& q_{r 0.5}(x)=0.0851 x+37.004 \\
& q_{r 1}(x)=0.0702 x+9.9796 \\
& q_{r 2}(x)=0.039 x+1.806 \\
& q_{r 4}(x)=0.0099 x+0.2478 \\
& q_{r 5}(x)=0.001 x+0.0023
\end{aligned}
$$




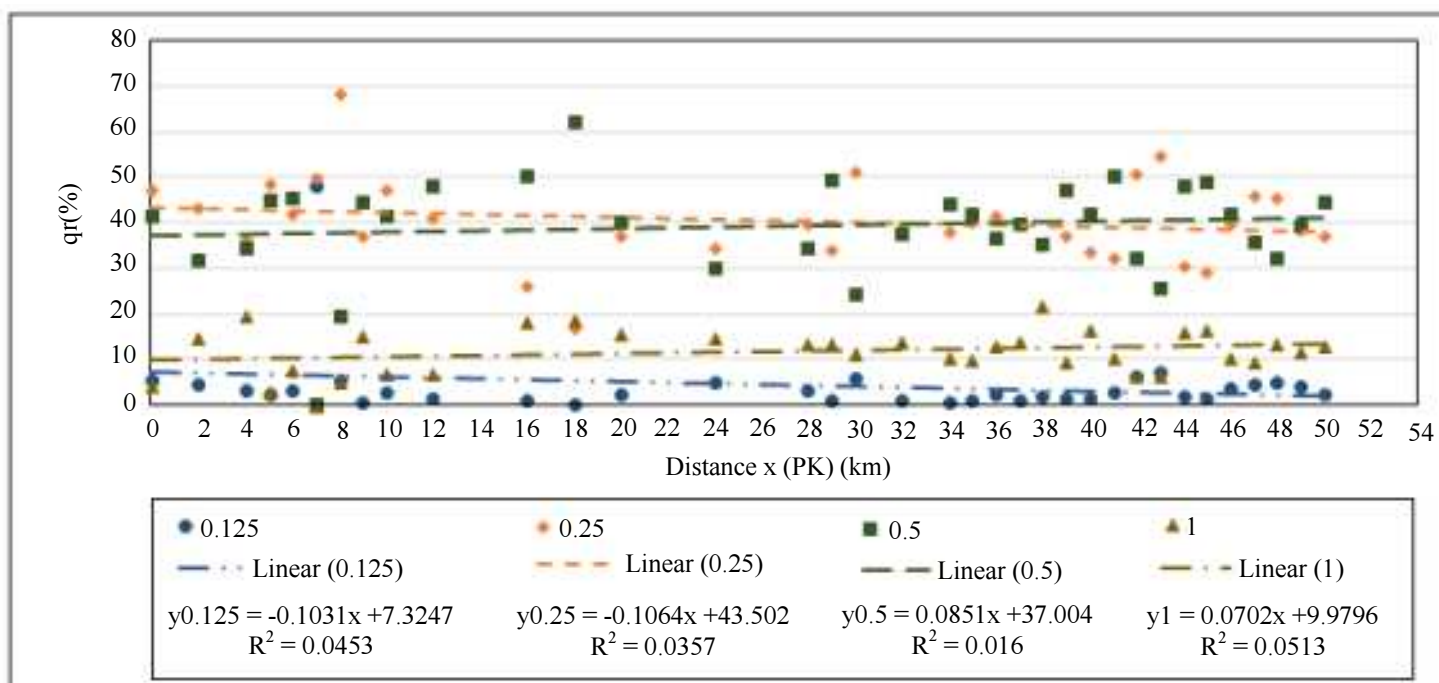

a. Differential distribution qr for $0.125-0.25-0.5-1$

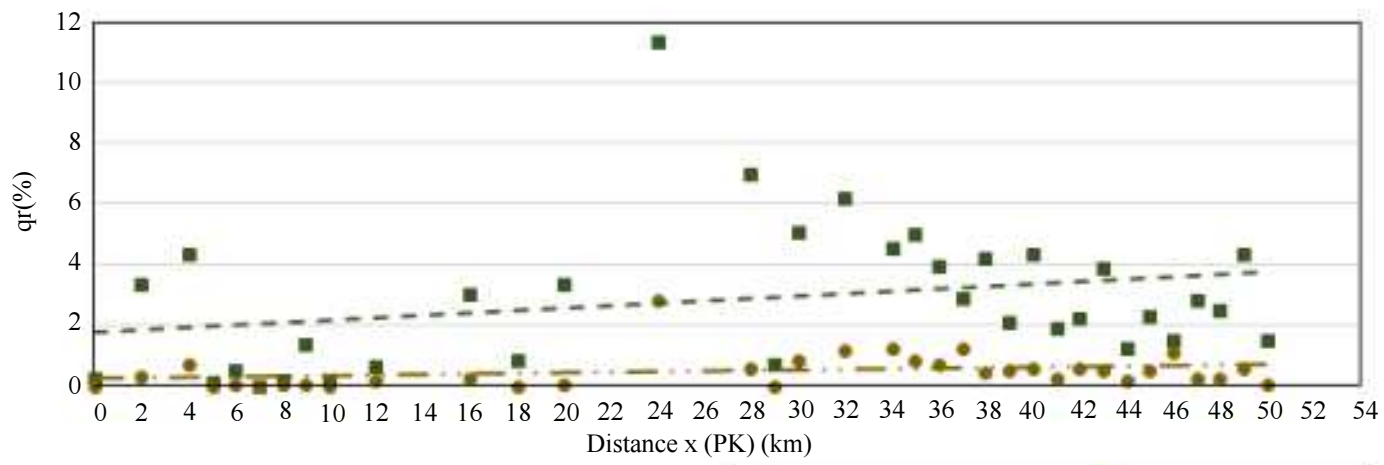

b. Differential distribution qr for 2-4

\begin{tabular}{|c|c|}
\hline$=2$ & $\bullet 4$ \\
\hline - - - Linear (2) & -... Linear (4) \\
\hline $\begin{array}{c}\mathrm{y} 2=0.039 \mathrm{x}+1.806 \\
\mathrm{R}^{2}=0.0713\end{array}$ & $\begin{array}{c}\mathrm{y} 4=0.0099 \mathrm{x}+0.2478 \\
\mathrm{R}^{2}=0.0812\end{array}$ \\
\hline
\end{tabular}

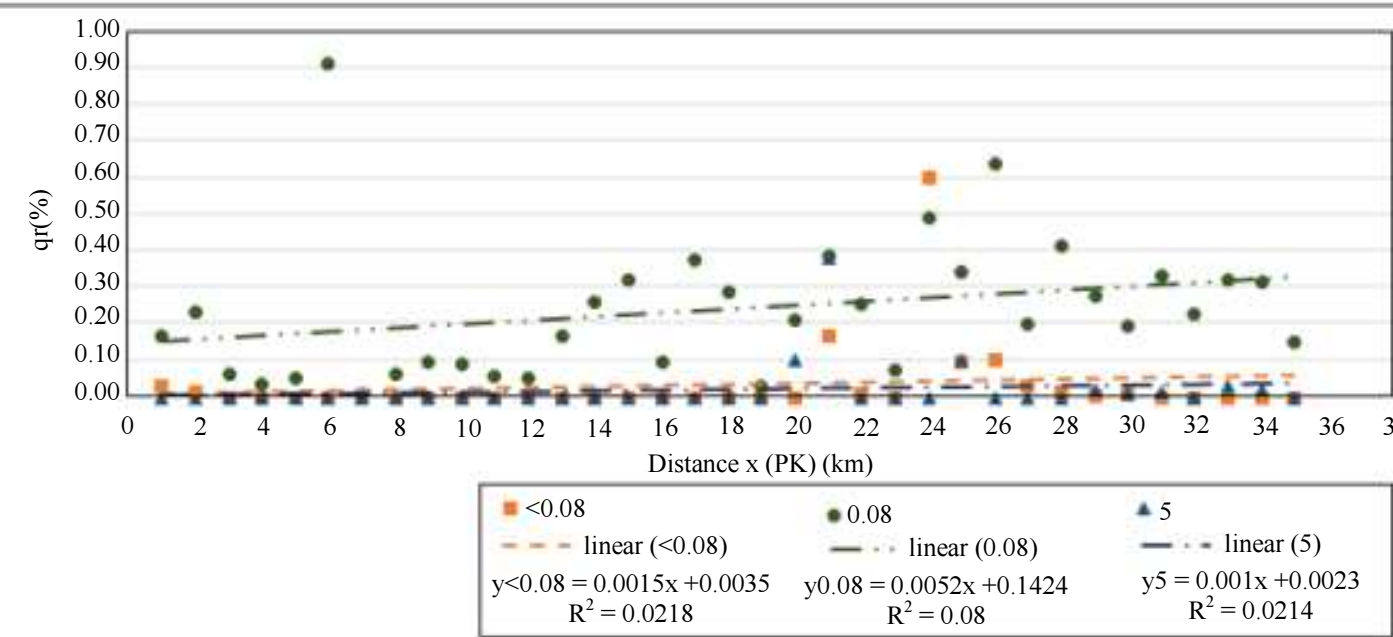

c. Differential distribution qr for " $<0.08$ " $-0.08-5$

Fig. 5: Differential distributions of the particle size according to the profiles 


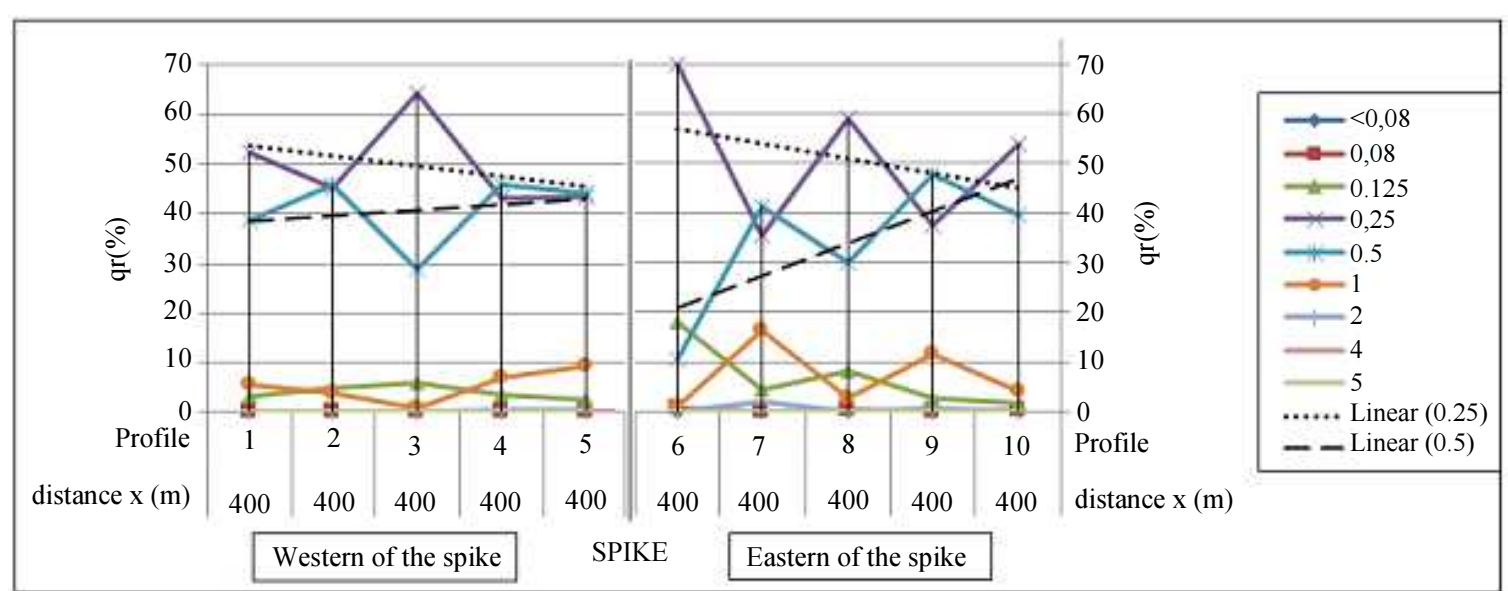

Fig. 6: Differential distributions of the grain size on each side of the spike E4

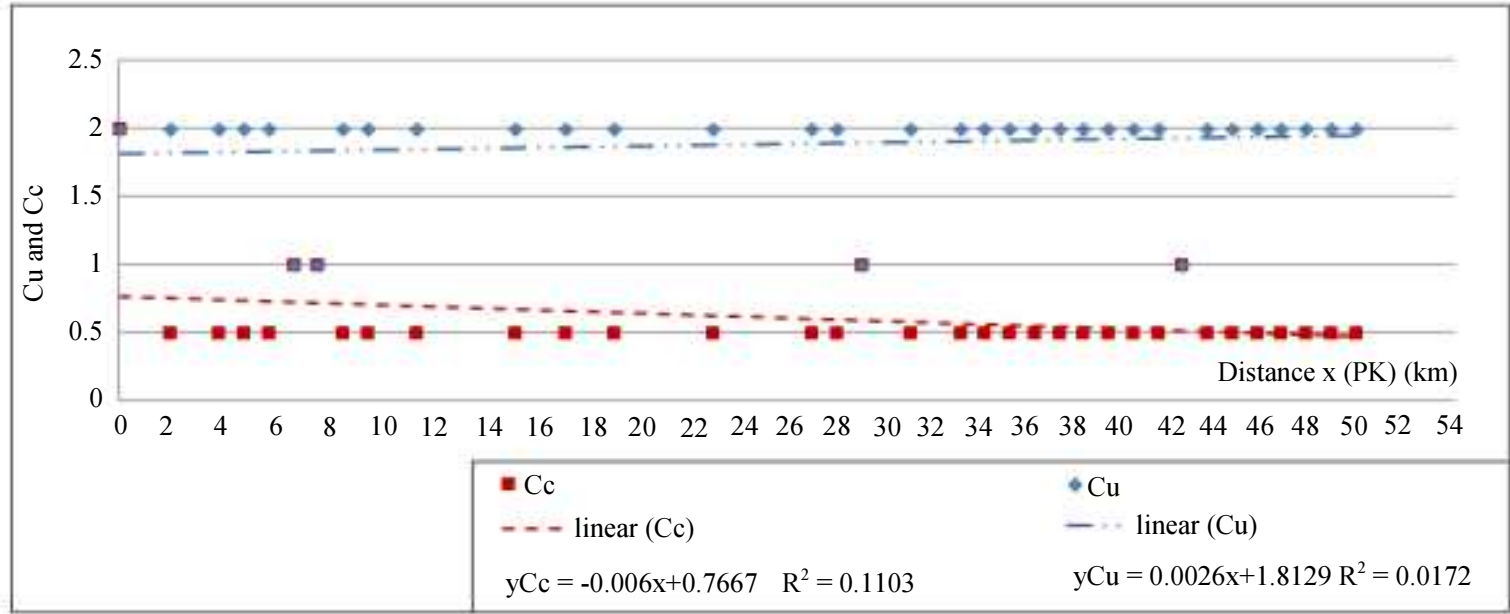

Fig. 7: Development of coefficients of uniformity $(\mathrm{Cu})$ and of bending $(\mathrm{Cc})$ according to profiles

In these equations $q_{r i}$ shows the differential distribution at sieve $i$ and $x$ the distance in $\mathrm{km}$ with regard to the point of reference called $P K O$ at the border Togo-Ghana:

- From Fig. 6 it is apparent that around the spike E4, whereas the sediments get rich in grains of $0.5 \mathrm{~mm}$ (growth of curves trends) following the direction of littoral drifting, those of $0.25 \mathrm{~mm}$ are getting poor in the sediments (decrease of trend curves). In the immediate vicinity of the spike E4, the grains of 0.5 $\mathrm{mm}$ are more important in the accretion zone than in the erosion zone. The reverse phenomenon happens for the grains of $0.25 \mathrm{~mm}$

\section{Classification of Sediments}

The different coefficients of uniformity and bendings of 35 profiles are given by Fig. 7 whereas those of each side of the spike E4 are given by Fig. 8. From this it is apparent that:
- $\quad$ The coefficients of uniformity $\left(C_{u}\right)$ are all equal to 2 except for KP7 (profile 6), PK8 (Profile 7), PK30 (profile 17) et PK43 (profile 28) that have a $C_{u}$ equal to 1 . Generally, this coefficient is increasing of PK 0 (profile 1) at KP50 (profile 35). As for coefficients of bending $\left(C_{c}\right)$, they are included between 0.5 and 1 . It presents an decreasing trend all along the littoral

This behaviour of coefficients can be illustrated by the smooth curves the equations of which are given by:

$$
\begin{aligned}
& C_{c}(x)=-0.006 x+0.7667 \\
& C_{u}(x)=0.0026 x+1.8129
\end{aligned}
$$

with:

$$
\begin{aligned}
C_{c} \text { and } C_{u}= & \text { Respectively the coefficients of } \\
& \text { bending and uniformity. } \\
x \quad & \text { Given as previously. }
\end{aligned}
$$


- On each side of the spike E4, the coefficient of uniformity is constant; as for the coefficient of bending, it is constant in the accretion zone, decreasing in the erosion zone

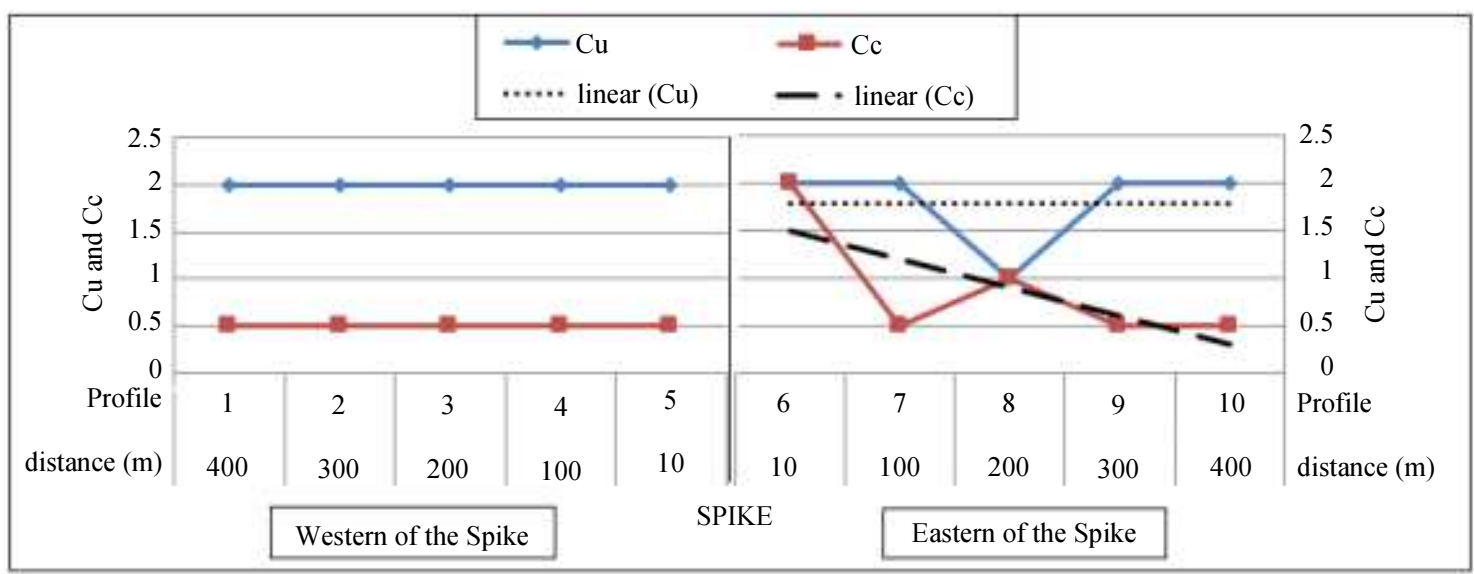

Fig. 8: Development of coefficients of uniformity $(\mathrm{Cu})$ and of bending $(\mathrm{Cc})$ on each side of the spike E4

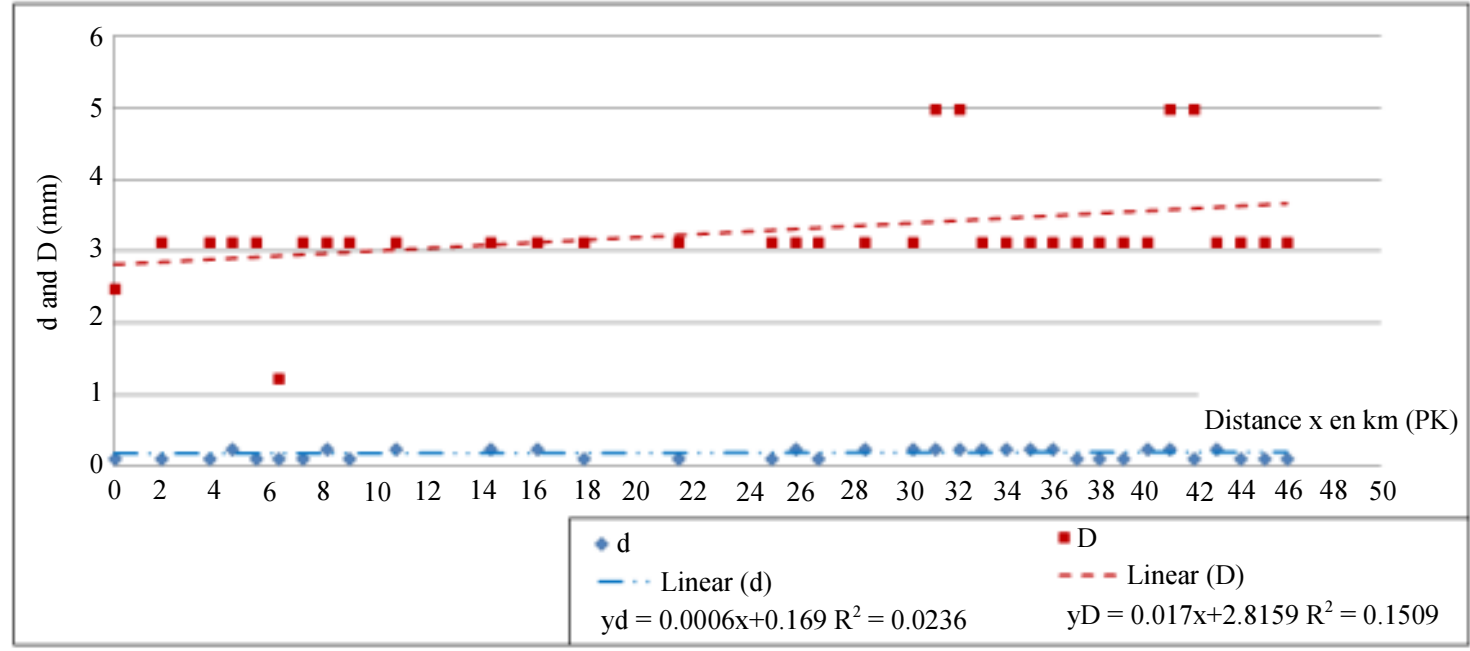

Fig. 9: Longitudinal distribution of $d$ and $D$

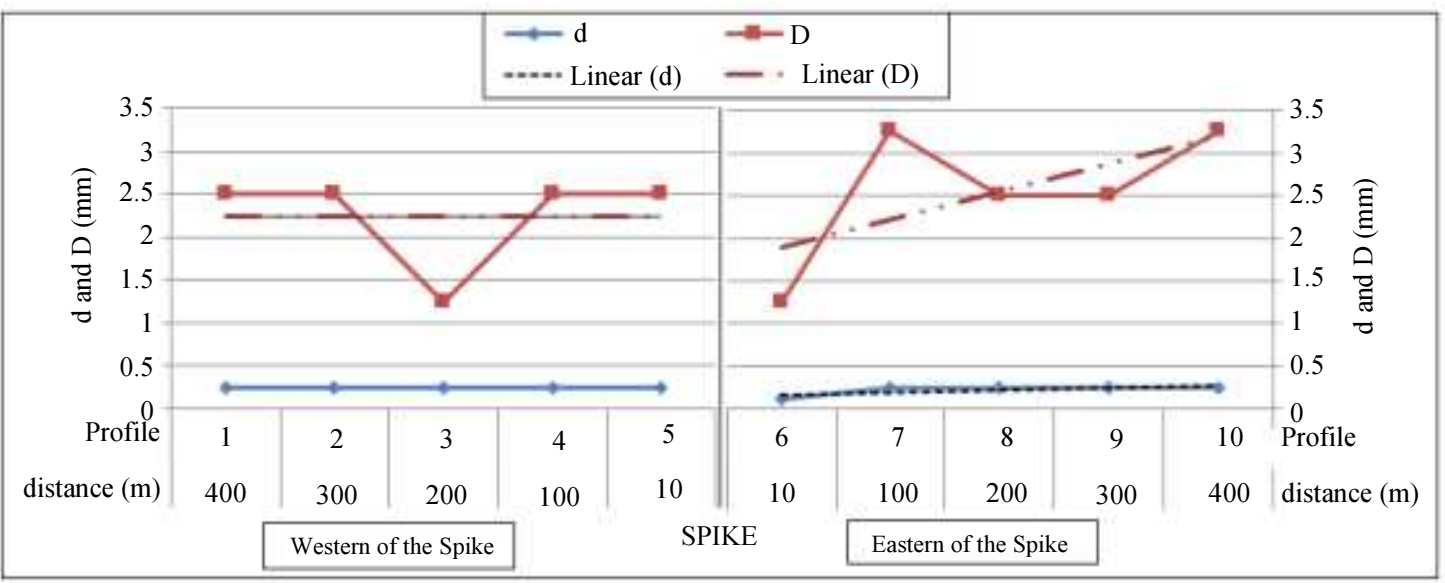

Fig. 10: Distribution of $d$ and $D$ on each side of the spike E4 


\section{Longitudinal Distribution of Granular Class}

Based on the system of Equation 5, the granular class of sediments by profile are calculated. Figure 9 and 10 provide the curves expressing the values $\mathrm{d}$ and $\mathrm{D}$ according to profiles. The curves of Fig. 9 and 10 show that:

- The smallest grain (d) is practically constant all along the profiles $(0.125 \mathrm{~mm}<\mathrm{d}<0.2 \mathrm{~mm})$. As for the biggest grain (D), it is constant $(\mathrm{D}=3.15 \mathrm{~mm})$ on all the profiles except for PK0 (profile 1) $(\mathrm{D}=2.5 \mathrm{~mm})$, at PK7 (profile 6) $(\mathrm{D}=1.25 \mathrm{~mm})$ and at PK 35, 36, 45 et 46 (profiles 20,21, 30 et 31) for which D is $5 \mathrm{~mm}$. But, in general it is increasing all along the littoral

This behaviour can be illustrated par the following smooth curves:

The smooth curves that illustrate this behaviour are given by the following equations:

$$
\begin{aligned}
& d(x)=0.0006 x+0.169 \\
& D(x)=0.017 x+2.8159
\end{aligned}
$$

with

$d$ and $D$ respectively the smallest and the biggest grains of sand

$x$ is given as previously.

- The smallest grain (d) is practically constant on each side of the spike. Whereas in the accretion zone the biggest grain (D) is constant, in the erosion zone, it is getting bigger and bigger as one is moving away from the spike

\section{Discussion}

It emerges from this study that sediments from the littoral are made up of grains that are getting bigger and bigger in the accretion zones (western of the spike) and erosion zone (eastern of the spike) in the direction of the littoral drifting. These sediments are less and less tight, badly graduated and more opened in the direction of the littoral drift. These observations are valid on the entire littoral for which the size of sediments increases in the direction of their transportation and gets richer in big particles of 0.5 $\mathrm{mm}, 1 \mathrm{~mm}, 2 \mathrm{~mm}$ and $4 \mathrm{~mm}$, with a more open size that is more and more badly graduated. In the immediate vicinity of the spike the grains are bigger in the accretion zone than in the erosion zone. This behavior of sediments is consistent with the results based on the study on the transversal development of differential distributions, the granular class (Amey, 2005) and the longitudinal distribution of mediumsized grains, sorting index and skweness (Amey, 2007) of Togolese littoral's sediments.

This highly tight grain size, which becomes less and less tight following the direction of the littoral drift and enriched in bigger particles, can be explained by the presence of natural works on the littoral that modifies the hydrodynamic behavour (Blivi, 1993) of sediments' particles.

In fact, the accretion zones of works receive waves with all their energy (important speediness) that carry sediments' elements. Which enables the rejection of big grains on the foreshore by transport. In the erosion zones, waves collide with the spike E4, lose their energies. The close downstream of the spike is then a water zone that is practically stagnant (Fig. 11). The biggest particles of the grains in this zone can no longer be transported by the low-energy waves. These particles settle then in the immerged beach. By going away from the work in the erosion zone, the low-energy waves carry the fine sand in suspension and medium sand by transport up to foreshore.

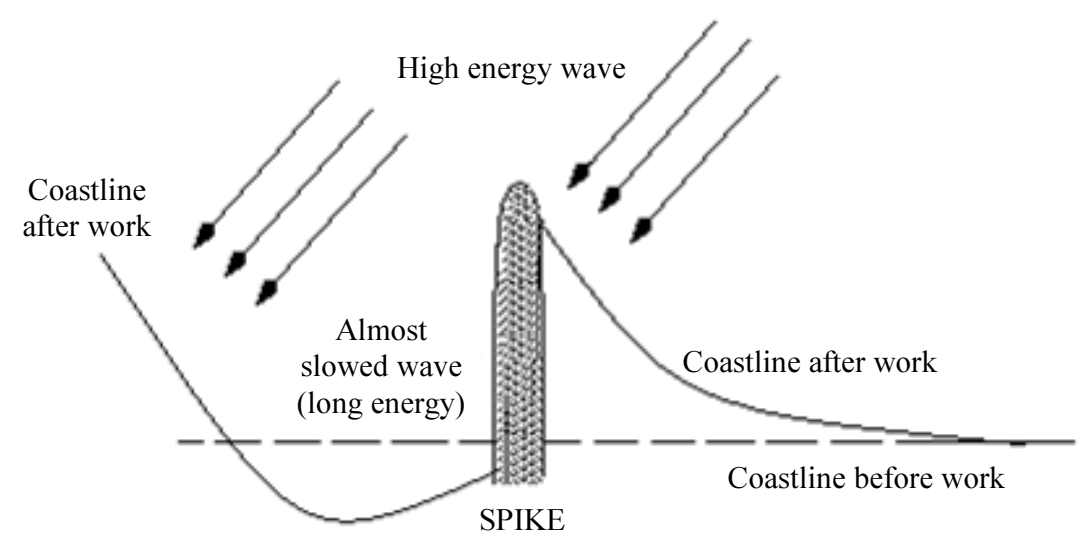

Beach

Fig. 11: Principle of sedimentary additions by waves at the level of spike E4 


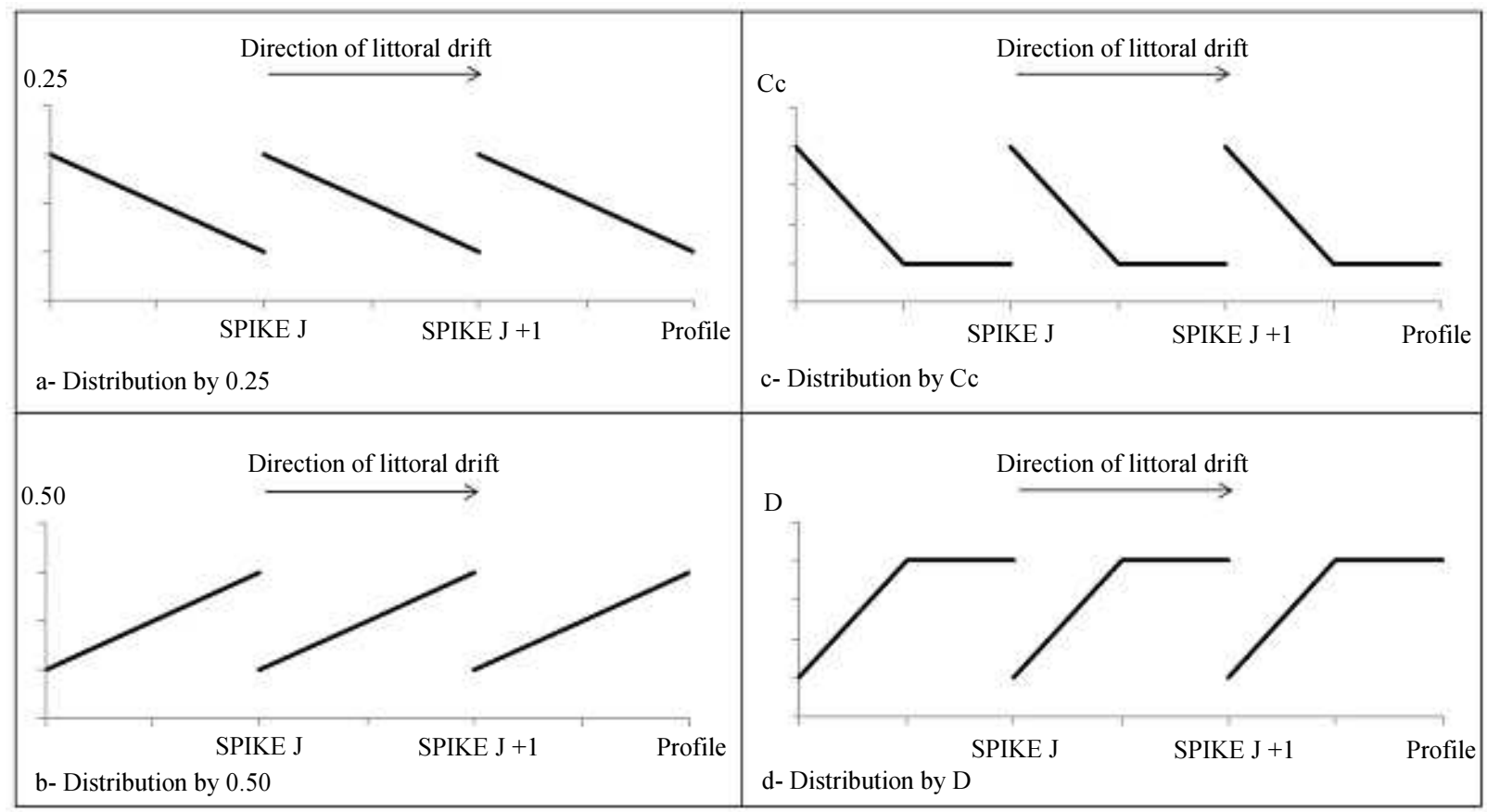

Fig. 12: Principle of distribution of sizes for a succession of the spike

The distribution of sediments 0.25 and $0.5 \mathrm{~mm}$, of coefficients of Hazen and of bending, of the granular class for a succession of spikes can then be illustrated by Fig. 12 .

\section{Conclusion}

From 210 samples collected all along the Togolese littoral and 10 on each side of the spike that are subjected to size analysis, it emerges that sediments of Togolese littoral are in general getting bigger and bigger in the direction of their transport from west to east (border Togo-Ghana and Togo-Benin). The spikes cause the creation of zones in erosion with fine sediments and of zones in accretion with big sediments. The sediments are sands of category " $f_{3}$ " with highly tight grain size, badly graduated $(\mathrm{Sm})$, that get increasingly rich in big grains and become more open and badly graduated in the direction of the littoral drift. The natural or artificial works along the Togolese littoral (Lomé harbor, Kpémé wharf, spikes and beach-rock) make it possible to get the following categories of sands on the Togolese littoral:

- Fine sands in the accretion zones far from works and in the erosion zones in the immediate vicinity of the work

- Medium-sized sands close to the works in the accretion zones and erosion zones

- Big-grain sands very close to the works in the accretion zones and far from the works in the erosion zones
As concrete materials, sands from Togolese littoral provide a huge granular potential:

- Medium-sized sands $(0.25$ and $0.5 \mathrm{~mm})$ and biggrain sands (over $1 \mathrm{~mm}$ ) are more appropriate for the construction of concretes the resistance of which is highly wanted and can be obtained from PK0 to PK50 except for PK7 and the accretion zones

- As for fine sands $(0.125$ and $0.25 \mathrm{~mm})$ they can be obtained in PK7 and in the erosion zones. They are wanted for coating works and for concrete the cladding aspect of which is very asked

- Because of its tight feature, the sand from Togolese littoral becomes a granulate providing a concrete that is less compact porous with a risk of segregation

\section{Acknowledgement}

This work could be done without formal logistic and financial support from FORMATEC we express our sincere gratitude to.

\section{Funding Information}

FORMATEC financed the research of materials and consumables necessary for the various tests.

\section{Author's Contributions}

Amey Kossi Bollanigni: Preparation of study samples, study realization, results processing and manuscript writing. 
Neglo Koumah: Project manager, conceptual contributions on the research.

\section{Ethics}

There are no ethical worries or concerns regarding this paper.

\section{References}

Aitcin, P.C., 1998. Notes de cours de technologie avancée des bétons. Université de Sherbrooke.

Amey, K.B., 2006, Caractérisation des sédiments sableux du littoral togolais: Expérimentation et détermination d'une formules de sables normal. Thèse de Doctorat Unique en Science de L'ingénieur.

Amey, K.B., K. Bedja and K. Neglo, 2005, Etude de l'évolution transversale des distributions différentielle et cumulative et de la classe granulaire des granulats marins du littoral togolais. J. Rech. Sci. Univ. Lomé (Togo), série E, 7: 13-20.

Amey, K.B., K. Bedja and K. Neglo, 2007. Distribution longitudinale de la granulométrie du sable du littoral togolais: Grain moyen, sorting index et skweness. J. Sci. Pour l'Ingénieur, 8: 1-8.
Blivi, A., 1993. Géomorphologie et dynamique actuelle du littoral du Golfe du Bénin (Afrique de l'Ouest). Thèse de Doctorat, Université Michel de Montaigne, Bordeaux.

Bresson, J., 1999, Granulats: identification classification. Fiche 111 de CERIB.

NF EN 12620, 2002. Granulats pour béton.

NF EN 933-1, 1997. Essais pour déterminer les caractéristiques géométriques des granulats, Partie 1: Détermination de la granularité - Analyse granulométrique par tamisage.

NF EN 933-2, 1996. Essais pour déterminer les caractéristiques géométriques des granulats, Partie 2: Détermination de la granularité-Tamis de contrôle, dimensions nominales des ouvertures.

NF ISO 9276-1, 1998. Représentation de données obtenues par analyse granulométrique - Partie 1: Représentation graphique.

Tchouani Nana, J.M. and M. Callaud, 2004. Cours de mécanique des sols: Tome I - Propriétés des sols. www.almohandiss.com. 